\title{
Top-Down and Bottom-Up Solutions Within One Mathematical Model on the Example of Energetics
}

\author{
Kulyk Mykhailo \\ Institute of General Energy NAS of Ukraine, Kyiv, Ukraine
}

Email address:

info@ienergy.kiev.com

To cite this article:

Kulyk Mykhailo. Top-Down and Bottom-Up Solutions Within One Mathematical Model on the Example of Energetics. American Journal of Electrical and Computer Engineering. Vol. 5, No. 1, 2021, pp. 25-31. doi: 10.11648/j.ajece.20210501.14

Received: March 1, 2021; Accepted: March 17, 2021; Published: March 26, 2021

\begin{abstract}
The TD-BU methodology covers a wide range of objects of various "physical" nature and with various analysis tasks. The feature of such objects is that they have a hierarchical structure. Mathematically, they are related by the formalization of upper-level processes by algebraic equations, while lower levels are described by means of mathematical programming. Currently being extensively researched the forecast status of ultra-large hierarchical systems such as "the country's economy - its fuel and energy complex" with certain requirements. The excessive dimensions of such systems create difficulties in their analysis in the classical formulation, so most researchers use diacoptic methods and therefore these tasks TD-BU are labor-intensive. There are a large number of objects to which the TD-BU methodology could be formally applied. We are talking, among other things, about forecasting the volume of production of all types of products, services and demand for them what is necessary for the activities of all sectors of the economy with details at hierarchical levels. For the tasks of this type the key is the problem of discrepancy of the upper and lower levels indicators. This problem cannot be solved by existing TD-BU models. This paper presents a mathematical model and methods for analytical determination of indicators of the upper and lower levels in the above problems, which solve the problem of ambiguity. The mathematical model is formed in such a way that provides an opportunity to find solutions for the upper and each of the lower (sectoral) levels in a unique, analytical form. Therefore, the search for solutions is non-iterative and not laborious. It is carried out in two stages. On the first of them, using known (standard) methods, forecasts are developed for preliminary indicators of the upper and lower levels. At the second stage a special system of algebraic equations is formed, from which analytical dependences for calculation of refined indicators of both levels are defined. This ensures a complete match between the upper indicator and the sum of the lower levels indicators, which is demonstrated by the example of forecasting electricity demand. These mathematical models and methods can also be used to reconcile the reporting indicators of the upper and lower levels of the respective objects (management structures, banks, trade network, etc.). Thus the coordinated decisions are formed in one stage.
\end{abstract}

Keywords: Top-Down, Bottom-Up, Iteration, Matrix, Discrepancy, Coincidence

\section{Introduction}

Both individual countries and humanity as a whole in all areas of their activities are constantly forced to solve various problems of planning and forecasting. As the depth of forecasting increases, its errors increase, which in the implementation of forecasting solutions can lead to significant losses. Concerning, forecasting methods are constantly evolving, which is especially important for the economy and energetics, the development of which is associated with large investments and even risks.

This study examines and improves a special group of forecasting methods that combine top-down and bottom-up approaches, abbreviated TD-BU. These methods apply only to systems and objects that have a hierarchical structure. Quite often, different hierarchical levels of such systems are described by different types of mathematical models, which complicates the mathematical relationships between levels.

The analysis of the literature sources, devoted to this problem, shows the presence of heterogeneous physical objects, systems, social or natural phenomena, etc., which require the use of this research apparatus. Characteristically, even in one area of knowledge, the problems solved using the TD-BU methodology require the use of multi-criteria models, 
and the criteria are often contradictory. This refers, first of all, to the problems of development and functioning of energy systems. In particular, some papers contain studies in which the optimization of power supply and heat supply systems was solved simultaneously with minimization of greenhouse gas emissions [1-3]. This forced the authors to use the simplest optimization methods, namely, purposeful sorting of options. Sometimes the problems of development of energy systems investigated together with the development of the economy according to compatible criteria for ensuring their reliable and economic functioning [4-6]. The compatibility of the criteria, in particular, enabled the authors to solve the problem in accordance with the theory of mathematical programming. It is noteworthy that in recent publications on energetics in terms of criteria factors of environmental protection and climate change come to the fore, what is the requirements of the time [7-11].

Important publications provide the theoretical foundations of the TD-BU methodology and typical examples of its application in production [12-15]. Here the model of creation process of operation strategy, which uses action plans of group of plants and directions of development of production strategy, is offered. A study of application this methodology in systems engineering, robotics and transport is presented.

Several noticeable publications consider important issues of development of the theory of TD-BU methodology and approaches to its effective familiarization and perfect study [16-19]. The methodological basis is the theory of testing and applied working-out in the development of problem-oriented software for training and coaching professionals in relevant fields.

\section{Formulation of the Problem}

As can be seen from the analysis of the literature, the TDBU methodology covers a very wide range of objects of different "physical" nature and with different analysis tasks. A characteristic feature of such objects is that they have a hierarchical structure. In addition, in mathematical terms, they are related by the formalization of upper-level processes, usually by algebraic equations and dependencies, while the lower levels are mostly described by means of mathematical programming. These publications actually investigate the forecast status and operation of ultra-large hierarchical systems such as "the country's economy - its fuel and energy complex" with certain requirements, in particular, on competitiveness and non-excess greenhouse gas emissions. The excessive dimensions of such systems create difficulties in their analysis in the classical formulation, so most researchers use methods of the system analysis in parts, using iterative procedures to reconcile intermediate indicators (diacoptic methods).

However, the analysis leads to the conclusion that the problem of this content is relevant. There are a large number of objects and a corresponding number of tasks to which the TD-BU methodology could be formally applied, but they remain unexplored and unsolved. We are talking, for example, about forecasting the demand for electricity for a certain future both for the country as a whole (upper level) and for all sectors of its economy with the social sphere inclusive (lower level). It is clear that the demand for electricity, determined by certain methods for the country as a whole, should be equal to the sum of the indicators calculated for each of its industries. However, in practice, such a coincidence is usually not observed, but there is a discrepancy between these data, the magnitude of which cannot satisfy designers or researchers. That is, for the tasks of this type the key is the problem of ambiguity of the upper and lower levels.

Such tasks are much more widespread than those currently solved using the classic TD-BU methodology. Indeed, forecasting the volume of production of all types of products, services and demand for them is necessary for the activities of all sectors of the economy with details on hierarchical levels, which is the basis for the functioning of markets and their stability.

But, as can be seen from the literature, this problem remains unresolved in its current state.

This paper presents a mathematical model and methods for analytical determination of upper and lower levels indicators in the above problems, which solve the problem of ambiguity. The mathematical model is formed in such a way that provides an opportunity to find solutions for the upper and each of the lower (sectoral) levels in a unique, analytical form. Therefore, the search for solutions is non-iterative. It is carried out in two stages. On the first of them, using known (standard) methods, preliminary forecasts are developed for indicators of the upper and lower levels. At the second stage a special system of algebraic equations is formed, from which analytical dependences for calculation of refined indicators of both levels are defined. This ensures a complete match between the upper indicator and the sum of the lower levels indicators.

These mathematical model and methods can be used quite reasonably to reconcile the reporting indicators of the upper and lower levels of the respective objects (management structures, banks, trade network, etc.). In this case the consensual decisions are formed in one stage.

\section{Mathematical Model}

The source information in this study is the vector of predictive functions $\boldsymbol{f}$, formed at a given period of time using certain mostly known forecasting methods

$$
\boldsymbol{f}=\left[f_{1}, f_{2}, f_{i}, f_{n}\right]^{\prime},
$$

where $f_{1}-$ Top-level forecast, $f_{i}, i=\overline{2-n}$ - Down-level forecasts.

According to the problem

$$
f_{1} \neq \sum_{i=2}^{n} f_{i}
$$


The purpose of the study in mathematical terms is to find a solution to the system of equations

$$
\begin{aligned}
& x_{a}=f_{1}, \\
& x_{2}=f_{2}, \\
& x_{i}=f_{i}, \\
& x_{n}=f_{n},
\end{aligned}
$$

in which $x_{a}$ is a $T$-level solution, and $x_{i}, i=\overline{2, n}-\mathrm{D}$-level solutions, and these solutions are related by an equation

$$
x_{a}-\sum_{i=2}^{n} x_{i}=0 .
$$

The system of equations (3) - (4) in the matrix-vector form is

$$
A x=F
$$

or

$$
\begin{aligned}
& 12 \boldsymbol{A} i n
\end{aligned}
$$

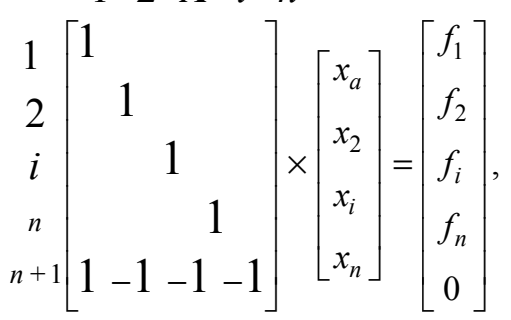

in which the vector $\left[f_{1}, f_{2}, f_{i}, f_{n}, 0\right]$ ' denote by $F$.

The system of equations (5), (6) has $n+1$ equation and $n$ unknowns. Such a system is redefined and therefore does not have an exact solution. To find the best of the approximate solutions, we use the Gaussian transformation in the form

$$
\boldsymbol{A}^{\prime} \boldsymbol{A} \boldsymbol{x}=\boldsymbol{A}^{\prime} \boldsymbol{F}
$$

For the right-hand side of equation (7), the dependence is valid

$$
\boldsymbol{A}^{\prime} \boldsymbol{F}=\boldsymbol{f}
$$

which is confirmed by multiplying $A^{\prime}$ by $F$.

Therefore, equation (7) will be considered in the form

$$
\boldsymbol{B} \boldsymbol{x}=\boldsymbol{f}, \boldsymbol{B}=\boldsymbol{A}^{\prime} \boldsymbol{A},
$$

in which

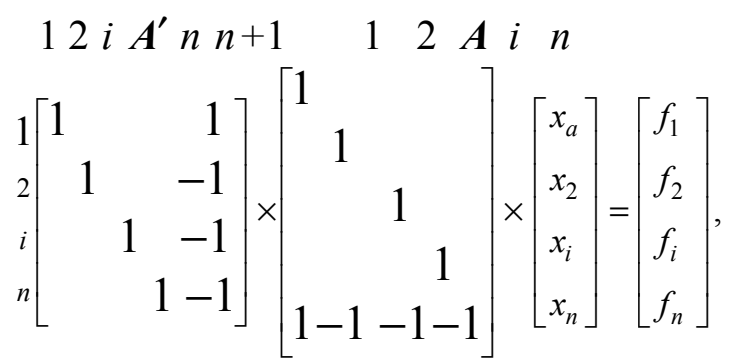

or, in the final version, the mathematical model of the problem has the form

$$
{ }_{i}\left[\begin{array}{ccccc}
1 & 2 & \boldsymbol{B} i & n \\
2 & -1 & -1 & -1 \\
-1 & 2 & 1 & 1 \\
-1 & 1 & 2 & 1 \\
-1 & 1 & 1 & 2
\end{array}\right] \times\left[\begin{array}{c}
x_{a} \\
x_{2} \\
x_{i} \\
x_{n}
\end{array}\right]=\left[\begin{array}{c}
f_{1} \\
f_{2} \\
f_{i} \\
f_{n}
\end{array}\right] .
$$

Analytical solutions. In algebraic equations (9), (10), the matrix $B$ is square with dimension $n$, the vectors $x$ and $f$ also have dimension $n$, the determinant $|B| \neq 0$, that is, the system of equations (9), (10) has one solution. This system has a structure that provides a unique opportunity to find this solution in an analytical form. To do this, we apply another Gaussian transformation to system (10), reducing the matrix $B$ to a triangular form and limiting itself to the dimension $n=$ 3. As a result, we obtain an algebraic system (11)

$$
\begin{aligned}
& 123 \\
& \left.\begin{array}{l}
1 \\
2 \\
3
\end{array} \quad \begin{array}{rrr}
2 & -1 & -1 \\
& & 1 \\
& & -8
\end{array}\right] \times\left[\begin{array}{l}
x_{a} \\
x_{2} \\
x_{3}
\end{array}\right]=\left[\begin{array}{l}
f_{1} \\
f_{1}+2 f_{2} \\
-2 f_{i}+2 f_{2}-6 f_{3}
\end{array}\right] \text {. }
\end{aligned}
$$

We find analytical solutions for the two dimensions of the system (11), namely, for $n=2$ and $n=3$.

For $n=2$ we have:

$$
\begin{aligned}
& \boldsymbol{x}_{\mathbf{2}}=\left(f_{1}+2 f_{2}\right) / 3=\boldsymbol{f}_{\mathbf{2}}+\left(\boldsymbol{f}_{\mathbf{1}}-\boldsymbol{f}_{\mathbf{2}}\right) / \mathbf{3}, \\
& 2 x_{a}=f_{1}+x_{2}=f_{1}+f_{2}+f_{1} / 3-f_{2} / 3, \\
& \boldsymbol{x}_{\boldsymbol{a}}=2 / 3 f_{1}-1 / 3 f_{2}=\boldsymbol{f}_{\mathbf{1}}-\left(\boldsymbol{f}_{\mathbf{1}}-\boldsymbol{f}_{\mathbf{2}}\right) / \mathbf{3} .
\end{aligned}
$$

For the case $n=3$ we receive:

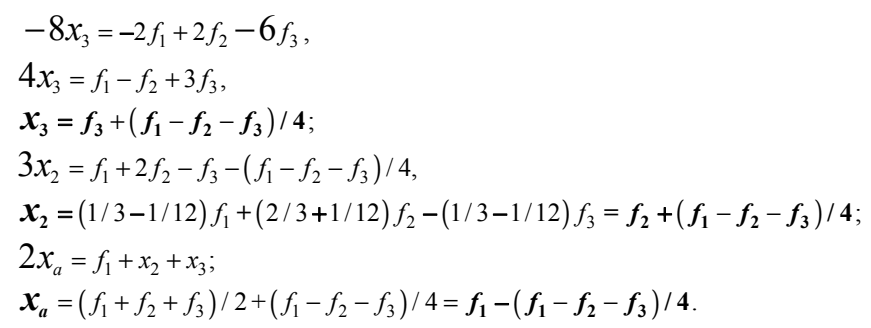

Analysis of the received decisions $x_{a}$ and $x_{i}, i=1,2$ for both cases shows that these solutions have the same structure, 
namely, the top-level solution has the form

$$
x_{a}=f_{1}-\frac{1}{n+1} r
$$

and sectoral decisions in this case take shape

$$
x_{i}=f_{i}+\frac{1}{n+1} r
$$

where

$$
r=f_{1}-\sum_{i=2}^{n} f_{i}
$$

is the difference between the upper level indicator and the sum of sectoral data,

$n$-dimension of the system (10), $i=\overline{2, n}$.

To confirm the dependences (12) - (14) we will show that they are valid not only for the dimension $\boldsymbol{n}$, but also for the dimension $\boldsymbol{n}+\mathbf{1}$. To do this, consider the structure (10) with dimension $\boldsymbol{n}+\mathbf{1}$, shown in scheme (15).

$$
\begin{aligned}
& \begin{array}{llllll}
1 & 2 & \boldsymbol{B} & i & n & n+1
\end{array}
\end{aligned}
$$

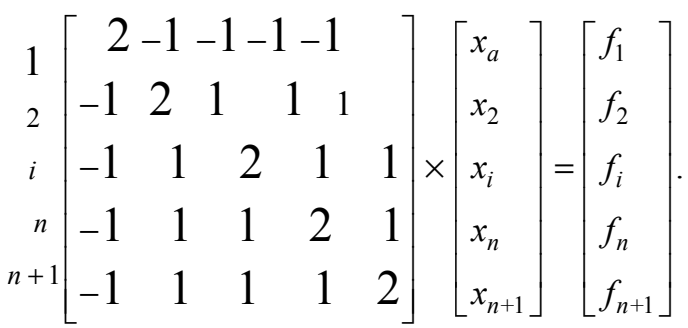

It is easily verified that the matrix $B$ in the system (15) has the structure of the matrix $B$ from equation (10) and differs only in the dimension.

Analytically determine the unknow $x_{a}$ and $x_{i}, i=\overline{2, n+1}$ in the system (15) taking into account the dependences (12) (14), in which the dimension $n$ is increased by one.

New unknown $x_{n+1}$ determined from the last equation of the system (15)

$$
2 x_{n+1}-x_{a}+\sum_{i=2}^{n} x_{i}=f_{n+1} \text {, }
$$

or

$$
2 x_{n+1}=f_{n+1}+f_{1}-r /(n+2)-\sum_{i=2}^{n} f_{i}-r(n-1) /(n+2) \text {. }
$$

Seeing that

$$
f_{1}-\sum_{i=2}^{n} f_{i}=r+f_{n+1}
$$

we get the result

$$
x_{n+1}=f_{n+1}+r /(n+2) .
$$

Unknown $x_{a}$ is determined from the first equation of the system (15)

$$
2 x_{a}-\sum_{i=2}^{n+1} x_{i}=f_{1}
$$

which, with considering (12) - (14) and (16), is transformed into a form

$$
2 x_{a}=f_{1}+\sum_{i=2}^{n+1} f_{i}+r n /(n+2)
$$

or

$$
2 x_{a}=2 f_{1}-r+r n /(n+2)
$$

and as a result

$$
x_{a}=f_{1}-r /(n+2)
$$

The dependence for $x_{i}$ in system (15) is determined from equation

$$
2 x_{i}-x_{a}+\sum_{k=2 ; k \neq i}^{n} x_{k}=f_{i}
$$

which is converted taking into account (12) - (14) and (16), (17) to the form

$$
2 x_{i}=f_{1}-\sum_{k=2 ; k \neq i}^{n+1} f_{k}-r n /(n+2)+f_{i}
$$

Applying the identity

$$
f_{1}-\sum_{k=2 ; k \neq i}^{n+1} f_{k}=r+f_{i}
$$

we get the final

$$
x_{i}=f_{i}+r /(n+2)
$$

Dependencies for $x_{m}, m=\overline{2, n}, \quad m \neq i$ are obtained analogously to (18) with insignificant differences in their definition, and as a result, taking into account (16), formula (18) is true for all $i=\overline{2, n+1}$.

The validity of the dependences (16) - (18) is also confirmed by their direct substitution into the system (15). In particular, for the equation $i$ have

$$
-f_{1}+r /(n+2)+\sum_{k=2}^{n+1} f_{k}+r n /(n+2)+f_{i}+r /(n+2)=f_{i} .
$$

Taking into account (14) we obtain the expression 


$$
-r+f_{i}+r(n+2) /(n+2)=f_{i}
$$

which is an identity.

Thus, the dependences (12) - (14) are the solution of the system of equations (10). However, their substitution in equation (4) does not satisfy him and gives an error

$$
x_{a}-\sum_{i=2}^{n} x_{i}=r /(n+1) \text {. }
$$

This is quite natural, because the system (3), (4) does not have, as noted, an exact solution.

However, it is noteworthy that the error $\boldsymbol{r}$ in the forecasts $f_{i}, i=\overline{1, n}$ due to transformations (7) - (9) in the system (10) decreases according to (19) by $n+1$ times. Therefore, it seems appropriate to organize the iterative process to ensure equation (4)

$$
\begin{aligned}
& B \boldsymbol{x}^{(1)}=f, B \boldsymbol{x}^{(2)}=\boldsymbol{x}^{(1)}, \ldots, \\
& B \boldsymbol{x}^{(m)}=\boldsymbol{x}^{(m-1)},\|\boldsymbol{x}\|^{(m-1)}-\|\boldsymbol{x}\|^{(m)} \leq \varepsilon,
\end{aligned}
$$

where $\quad-$ permissible error, $m$-number of iterations.

Decision $x^{(1)}$ after the first iteration in the form (12) - (14) already found. After the second iteration, it looks like

$$
\begin{aligned}
& \boldsymbol{x}_{a}^{(2)}=f_{1}-\left(\frac{1}{n+1}+\frac{1}{(n+1)^{2}}\right) \boldsymbol{r}, \\
& \boldsymbol{x}_{i}^{(2)}=f_{i}+\left(\frac{1}{n+1}+\frac{1}{(n+1)^{2}}\right) \boldsymbol{r}, i=\overline{2, n} .
\end{aligned}
$$

The solution of the iterative process (20) for the $m$-th iteration is determined using the method of complete induction. Considering (21), there is reason to assert that after the $m$-th iteration process (20) will provide a solution

$$
\begin{gathered}
\boldsymbol{x}_{\boldsymbol{a}}^{(\boldsymbol{m})}=\boldsymbol{f}_{\mathbf{1}}-s(m) \boldsymbol{r}, \\
\boldsymbol{x}_{\boldsymbol{i}}^{(\boldsymbol{m})}=\boldsymbol{f}_{\boldsymbol{i}}+s(m) \boldsymbol{r}, i=\overline{2, n}, \\
S(m)=\sum_{k=1}^{m} 1 /(n+1)^{k} .
\end{gathered}
$$

According to the method of complete induction, the solution after the $m+1$ iteration should have the same form as in (22) - (24) with the difference that in it instead of the value of $m$ will appear $m+1$.

According to (12) - (14) and using (20), (22) - (24), the solution after the $m+1$ iteration is represented in the form

$$
\boldsymbol{x}_{\boldsymbol{a}}^{(\boldsymbol{m}+\mathbf{1})}=\boldsymbol{x}_{\boldsymbol{a}}^{(\boldsymbol{m})}-\frac{1}{n+1}\left(\boldsymbol{x}_{\boldsymbol{a}}^{(\boldsymbol{m})}-\sum_{i=2}^{n} x_{i}^{(m)}\right)
$$

$$
\boldsymbol{x}_{i}^{(\boldsymbol{m}+\mathbf{1})}=\boldsymbol{x}_{\boldsymbol{i}}^{(\boldsymbol{m})}+\frac{1}{n+1}\left(\boldsymbol{x}_{\boldsymbol{a}}^{(\boldsymbol{m})}-\sum_{i=2}^{n} x_{i}^{(m)}\right)
$$

Dependence (25) is revealed using (22) - (24):

$$
\begin{aligned}
& \boldsymbol{x}_{\boldsymbol{a}}^{(\boldsymbol{m}+\mathbf{1})}=f_{1}-s(m) \boldsymbol{r}-(1 /(n+1)) \times \\
& \times\left(f_{1}-s(m) \boldsymbol{r}-\sum_{i=2}^{n}\left(f_{i}+s(m) \boldsymbol{r}\right)\right)= \\
& =f_{1}-r /(n+1)-(1-1 /(n+1)-(n-1) /(n+1)) s(m) \boldsymbol{r} .
\end{aligned}
$$

Seeing that

$$
(1-1 /(n+1)-(n-1) /(n+1))=1 /(n+1)
$$

and

$$
S(m) /(n+1)=\sum_{k=2}^{m+1} 1 /(n+1)^{k}
$$

dependence (27) is transformed into form

$$
\boldsymbol{x}_{\boldsymbol{a}}^{(\boldsymbol{m}+1)}=f_{1}-s(m+1) r
$$

which is according to the method of complete induction and confirms correctness of (22). The correctness of the dependence $(23)$ is proved similarly.

To further use solutions (22), (23) it is necessary to determine the sum (24) with an unlimited increase in the number of iterations $m$, i.e., it is necessary to establish a convergence limit

$$
c(n)=\lim _{m \rightarrow \infty} s(m)=\lim _{m \rightarrow \infty} \sum_{k=1}^{m}\left(1 /(n+1)^{k}\right) .
$$

Series $\sum_{k=1}^{m}\left(1 /(n+1)^{k}\right)$ coincides with all the attribute.

The limit of convergence is determined by the assumption with its subsequent verification. Suppose that such a limit is a quantity

$$
c(n)=1 / n \text {. }
$$

This assumption is justified, in particular, by the fact that the amount

$$
\sum_{k=1}^{4} 1 /(n+1)^{k}=0,0999931 \text { at } n=10 \text {. }
$$

To prove (29) we form according to (24) the difference between $c(n)$ and the partial sum $s(m)$, which at $m \rightarrow \infty$ must turn into zero

$$
p(m)=c(n)-s(m)
$$

Value $p(m)$ at $m=1$ is equal to $p(1)=1 /(n(n+1))$ and at 
$m=2 p(2)=1 /\left(n(n+1)^{2}\right)$.

By the method of complete induction we assume that the value of $p(m)$ has the form

$$
p(m)=1 /\left(n(n+1)^{m}\right)
$$

and prove that this expression is valid for a series with $m+1$ members, i.e,

$$
p(m+1)=1 /\left(n(n+1)^{m+1}\right) .
$$

Denote $n+1=\omega$ then according to (30) we establish

$$
p(m+1)=\left(\omega^{m+1}-\left((\omega-1) \sum_{k=0}^{m} \omega^{k}\right)\right) /\left((\omega-1) \omega^{m+1}\right) .
$$

Revealing the amount $\sum_{k=0}^{m} \omega^{k}$, we receive

$$
(\omega-1) \sum_{k=0}^{m} \omega^{k}=\omega^{m+1}-1
$$

As a result

$$
p(m+1)=\left(1 /(\omega-1) \omega^{m+1}\right)=1 / n(n+1)^{m+1} .
$$

This dependence proves that expression (31) is valid for all positive integers $m$. In this regard $\lim _{m \rightarrow \infty} p(m)=\lim _{m \rightarrow \infty} 1 /(n+1)^{m}=0$, that is, the dependence (29) is true. Thus, the dependence (31) is valid for all positive integer $m$. Then $\lim _{m \rightarrow \infty} p(m)=\lim _{m \rightarrow \infty} \frac{1}{n(n+1)^{m}}=0$ at all positive integers $n$ and $m$, and the dependence (21) is true.

The consequence of this is that the dependences (22), (23) take the form

$$
\begin{gathered}
x_{a}=f_{1}-r / n, \\
x_{i}=f_{i}+r / n, i=\overline{2, n} .
\end{gathered}
$$

Substitution of dependences (33), (34) satisfies equation (4).

\section{Example}

Dependencies (33), (34) provide an opportunity for practical application of the developed methodology to reconcile contradictory Top- and Down forecasts. The table shows the results of the refinement of the demand forecast for electricity in Ukraine at the level of 2030 year. Demand was forecast using macroeconomic indicators (electricity capacity and gross domestic product, T-level) and by determining demand in sectors of the economy based on the electricity capacity of sectoral production and its volumes. These indicators were determined using methods of detecting dependencies. For the Population sector, demand was determined using extrapolation methods of the respective power consumption series. These forecasts are presented in the table in the column "Before agreement", which contains a consolidated macro-forecast for country as a whole (level Top) and forecasts for 4 sectors and their sum (level Down). The difference in the indicators of T- and D-levels is quite significant and is $r=41.2 \mathrm{TWh}$.

Table 1. Forecast of demand (2030 year) for electricity in Ukraine using the TD-BU model (TWh).

\begin{tabular}{llll}
\hline $\begin{array}{l}\text { № } \\
\mathbf{p} / \mathbf{p}\end{array}$ & Indicator Sector & $\begin{array}{l}\text { Before } \\
\text { agreement }\end{array}$ & $\begin{array}{l}\text { After application } \\
\text { TD-BU }\end{array}$ \\
\hline & Level Top & 198,5 & 190,26 \\
& Level Down & 157,3 & 190,26 \\
1 & Metallurgical and fuel industry & 41,9 & 50,14 \\
2 & Other industry & 31,2 & 39,44 \\
3 & Agriculture, services, transport, & 39,4 & 47,64 \\
4 & other consumers & 44,8 & 53,08 \\
\hline
\end{tabular}

Using the described methods, calculations of electricity demand volumes (column "After application of TD-BU" in the table) were performed, which ensured complete coincidence of T- and D-levels.

\section{Conclusions}

From the analysis of the literature, it follows that the TDBU methodology covers a wide range of objects of various "physical" nature and with various tasks of analysis. A characteristic feature of such objects is that they have a hierarchical structure. In addition, in mathematical terms, they are related by the formalization of upper-level processes, usually by algebraic equations and dependencies, while the lower levels are mostly described by means of mathematical programming. These publications actually investigate the forecast status and operation of ultra-large hierarchical systems such as "the country's economy - its fuel and energy complex" with certain requirements, in particular, on competitiveness and non-excess greenhouse gas emissions. The excessive dimensions of such systems create difficulties in their analysis in the classical formulation, so most researchers use methods of analysis of the system in parts using iterative procedures to reconcile intermediate indicators (diacoptic methods). Therefore, these tasks TD-BU are laborintensive.

At the same time, the analysis leads to the conclusion that the problem of other content is relevant. There are a large number of objects and a corresponding number of tasks to which the TD-BU methodology could be formally applied, but they remain unexplored and unsolved. We are talking, among other things, about forecasting the demand for electricity for a certain future both for the country as a whole (upper level) and for all sectors of its economy, including the social sphere (lower level). The demand for electricity, 
determined by certain methods for the country as a whole, should be equal to the sum of the indicators calculated for each of its industries. However, in practice, such a coincidence is usually not observed, but there is a discrepancy between these data, the magnitude of which can not satisfy designers or researchers. That is, for the tasks of this type the key is the problem of ambiguity of the upper and lower levels. This problem cannot be solved by existing TDBU models.

Such tasks are much more widespread than those currently solved using this methodology. Forecasting the volume of production of all products types, services and demand for them is necessary for the activities of all sectors of the economy with details at hierarchical levels, which is the basis for the functioning of markets and their stability. But, as can be seen from the literature, this problem remains unresolved in its current state.

This paper presents a mathematical model and methods for analytical determination of upper and lower levels indicators in the above problems, which solve the problem of ambiguity. The mathematical model is formed in such a way that provides an opportunity to find solutions for the upper and each of the lower (sectoral) levels in a unique, analytical form. Therefore, the search for solutions is non-iterative and not laborious. It is carried out in two stages. On the first of them, using known (standard) methods, forecasts are developed for indicators of the upper and lower levels. At the second stage a special system of algebraic equations is formed, from which analytical dependences for calculation of indicators of both levels are defined. This ensures a complete match between the upper indicator and the sum of the lower levels indicators.

These mathematical models and methods can also be used to reconcile the reporting indicators of the upper and lower levels of the respective objects (management structures, banks, trade network, etc.). Wherein the coordinated decisions are formed in one stage.

\section{References}

[1] Jacobsen H. K. (1998) Integrating the bottom-up and topdown approach to energy - economy modelling: the case of Denmark. Energy Economics, 20, 443-461.

[2] Rivers N. \& Jaccard M. (2005) Combining Top-Down and Bottom-Up Approaches To Energy-Economy Modeling Using Discrete Choice Methods. The Energy Journal, 26 (1), 83-106.

[3] C. Böhringer C. (1998) "he Synthesis of Bottom-Up and TopDown in Energy Policy Modeling. Energy Economics, 20 (3), 233-248.

[4] Frei C. W., Hadi P.-A. \& Sarlos G. (2003) Dynamic formulation of a top-down and bottom-up merging energy policy model. Energy Policy, 31 (10), 1017-1031.
[5] Böhringer C. \& Rutherford T. F. (2008) Combining bottom-up and top-down. Energy Economics, 30 (2), 574-596.

[6] Helgesen P. J. (2013) Top-down and Bottom-up: Combining energy system models and macroeconomic general equilibrium models. CenSES working paper 1/2013, [online]. Available: http://www.ntnu.no/censes/working-papers.

[7] Murphy R. N., Rivers N. \& Jaccard M. (2007) Hybrid modeling of industrial energy consumption and greenhouse gas emissions with an application to Canada. Energy Economics, 29 (4), 826-846

[8] Strachan N. \& Kannan R. (2008) Hybrid modelling of longterm carbon reduction scenarios for the UK. Energy Economics, 30 (6), 2947-2963.

[9] Etiope G. \& Schwietzke S. (2019) Global geological methane emissions: An update of top-down and bottom-up estimates. Elementa: Science of the Anthropocene, 7: 47. https://doi.org/10.1525/elementa.383.

[10] Bottom-Up and Top-Down Approaches for National MRV Systems. (2018) UNDP's NDC Support Programme is funded by the European Union and the governments of Germany and Spain as a contribution to the NDC Partnership.

[11] MRV in Practice -connecting Bottom-Up and Top-Down Approaches for developing National MRV systems for NDCS. (2018), NDC Support Programme.

[12] Yoon Hee Kim, Sting F. J. \& C. H. Loch C. H. (2014) TopDown, Bottom-Up, or Both? Toward an Integrative Perspective on Operations Strategy Formation In Press, Journal of Operations Management. DOI: 10.1016/j.jom.2014.09.005.

[13] Crespi V., Galstyan A. \& Lerman K. Top-down vs bottom-up methodologies in multi-agent system design., Reasech Gate. DOI: $10.1007 / \mathrm{s} 10514-007-9080-5$.

[14] P. M. Swamidass P. M., Darlow N. \& Baines T. (2001) Evolving Forms of Manufacturing Strategy Development: Evidence and Implications. International Journal of Operations \& Production Management, 21, 1289-1304.

[15] Kulmer V. (2012) Directed Technological Change in a Bottom-Up/Top-Down CGE model: Analysis of Passenger Transport. Ecomod Conference Paper, [online]. Available: ecomod.net $>$ system $>$ files $>$ Kulmer_Veronika_Direct.

[16] Yin R. K. (2009) Case Study Reserach: Design and Methods. 4th ed., Sage, Thousand Oaks, CA.

[17] Ammann P. \& Offutt J. (2008) Introduction to Software Testing. Cambridge University Press, New York.

[18] Rutherford T. F. (2005) Integrating Bottom-Up into Top-Down: A Mixed Complementarity Approach. Discussion Paper No. 05-28, ZEW, Mannheim.

[19] Martinsen T. (2011) Introducing technology learning for energy technologies in a national CGE model through soft links to global and national energy models. Energy Policy, 39 (6), 3327-3336. 